\title{
Optimized Multi-Event Simultaneous Departure Routes for Major Hub Airport
}

\author{
Zhang Song, H. G. Visser, Hou Mingshan, and Cong Wei
}

\begin{abstract}
This study focuses on developing a multi-event simultaneous departure routing for the Amsterdam Airport Schiphol. The aircraft ground tracks are shaped by rational Bezier curve, and the trajectory optimization problem is reduced to a constrained parametric optimization formulation with few unknown optimizers. In order to handle the constraints, the continuous tracks are discretized into a series of data points and approximated by piecewise linear curves. A data-points selection strategy is introduced to improve the calculation efficiency and guarantee the feasibility of the optimized track. The original constrained parameter optimization problem is transformed to an unconstrained one using a penalty function method and subsequently solved by a genetic algorithm and pattern search method. The details of penalty function formulation are given in this study. The feasibility of the proposed method is demonstrated in numerical examples. The results show that both the number of people highly annoyed and track distance are decreased in simultaneous optimization relative to a scenario where departure routes are optimized in isolation.
\end{abstract}

Index Terms-Trajectory optimization, route shaping, multi-event simultaneous route, rational bezier curve.

\section{INTRODUCTION}

The growth in the demand for civil aircraft movements has already proven a challenge for major airports in recent years. This challenge is even further enhanced by the continuously increasing opposition of local communities against the noise annoyance generated by landing and departing aircraft. Amsterdam Airport Schiphol (AMS) for example - the fifth largest airport by aircraft movements in Europe accommodated 440,000 movements in 2007 compliant with noise regulations to ensure acceptable noise exposure levels for near-airport residents. This is opposed, however, by a projected growth to 510,000 movements by 2020 [1]. One of the approaches to accommodate this projected growth is to design noise friendly departure and arrival routes and procedures.

Whereas research into noise abatement departure and arrival procedures has been quite extensive in recent decades, the development of community noise abatement routes has been less common. Erkelens [2] proposed the use of Area Navigation (RNAV) to design Precision Navigation

Manuscript received June 24, 2014; revised August 21, 2014.

Zhang Song and Hou Mingshan are with the Northwestern Polytechnical University, Xi'an, China (e-mail: zhangsong.gz@ gmail.com, mingshan@nwpu.edu.cn).

H. G. Visser is with Delft University of Techology, Delft, Netherland (e-mail: h.g.visser@tudelft.nl).

Cong Wei is with the Nanjing University of Aeronautics and Astronautics, Nanjing, China (e-mail: W.Cong-1@tudelft.nl).
Instrument Departures (PNID) to reduce the flight track dispersion and allow for more lateral freedom in trajectory design. Focusing on both procedures and routing, Prats et al. [3] applied 4D optimization techniques in an effort to numerically minimize the noise impact in a number of discrete points. In References [4]-[7] noise-optimized approach and departure trajectories are computed based on a direct numerical optimization techniques that enables to generate (piecewise) continuous optimal trajectories for a point-mass modeled fixed-wing aircraft. The numerical tool that has been used in these particular studies is called NOISHHH. Considering cumulative noise criteria, Braakenburg et al. [8] developed a multi-event trajectory optimization methodology based on NOISHHH, which allows to synthesize RNAV approach routes that minimize a cumulative measure of noise.

Quite extensive previous research has mainly focused on designing a single route (in isolation) only. Due to their mutual dependence, multiple routes therefore now need to be optimized sequentially in an iterative fashion, taking the legal criteria for noise nuisance and regulations for air navigation services into account. The required calculation time is excessive, while convergence of the design cannot be guaranteed. Clearly, this points in the direction of the development of new efficient methodology that can optimize multiple dependent routes simultaneously.

This study aims to optimize multi-event simultaneous departure routing at a large international airport. The primary goal is to optimally shape the shared ground track, assuming given procedures (altitude and speed profiles as functions of distance to runway) for the various aircraft types that visit Amsterdam Airport Schiphol within the considered operational period.

In this paper, we first formulate the design of a community noise optimized approach route as a multi-event trajectory optimization problem. Next, a brief discussion on the employed trajectory optimization technique is presented. The overall approach is then demonstrated in numerical examples involving the design of community noise optimized departure route for the Kaagbaan and Aalsmbeerbaan runways South-outbound of Amsterdam Airport Schiphol.

\section{Multi-Event Trajectory Optimization FORMULATION}

\section{A. Aircraft Dynamics}

The aircraft trajectory optimization framework developed in this study relies on a point-mass dynamic model formulation. With the vertical profiles prescribed for each aircraft type, the trajectory optimization problem in essence 
only needs to address the ground track kinematics shared by all aircraft. Using along-track distance $\mathrm{s}$ as the independent variable, and assuming small flight path angle $\gamma$ such that $\cos \gamma=1$, the kinematic equations can be written as [8]:

$$
\begin{aligned}
& \frac{d x}{d s}=\cos \chi \\
& \frac{d y}{d s}=\sin \chi \\
& \frac{d \chi}{d s}=u
\end{aligned}
$$

where $\chi$ is the heading angle and $x$ and $y$ define the position coordinates in a Cartesian reference frame. The heading rate $u$ is the inverse of the turn radius $R$, and is chosen as the control variable in the optimal control formulation. By selecting the heading rate rather than the heading angle as control variable, constraints regarding bank angles, turn rates and turn radii can be more easily imposed.

With the kinematic equations now defined for all aircraft types on a single route, what remains to be determined are the input parameters required for the noise modeling. Since the airspeed $V(s)$ and the altitude $h(s)$ can be found from the prescribed procedures, and the heading rate $u(s)$ is known from the optimal control history, first the bank angle is determined using:

$$
\mu=\arctan \left(u \frac{V^{2}}{g_{0}}\right)
$$

This can then in turn be used to determine the required thrust:

$$
T=V \frac{d V}{d s} \frac{W}{g_{0}}+C_{D} \frac{1}{2} \rho V^{2} S+W \frac{d h}{d s}
$$

where $C_{D}$ is the drag coefficient and $W$ is the aircraft weight.

In addition to modeling of the trajectory dynamics, in order to obtain more realistic results, a number of operational constraints are imposed. Since airspeed and altitude constraints are already accounted for in the prescribed altitude and airspeed profiles, these constraints only pertain heading changes in the ground track. First, a maximum turn rate constraint is imposed. Note that with the turn radius $R$ and the airspeed derived from the prescribed procedures, the turn rate can be determined from:

$$
\frac{d \chi}{d t}=\frac{d \chi}{d s} \frac{d s}{d t}=\frac{V}{R}=u V
$$

Then, the turn rate constraint can be defined as:

$$
|u| \leq\left.\frac{d \chi}{d t}\right|_{\max } / V
$$

In addition, a bank angle constraint is imposed using Eq. 4:

$$
|u| \leq \frac{g_{0} \tan \left(\mu_{\max }\right)}{V^{2}}
$$

It is noted that the constraints in Eq. 7 and Eq. 8 are both dependent on the airspeed, which varies for each representative aircraft type. As a result, the aircraft with the highest airspeed at a given location $s$ determines the limiting value of the control value $u$.

\section{B. Community Noise Impact Optimization}

With the position, airspeed and thrust for each representative aircraft type known, all variables required for the noise modeling are now available. For the acoustic calculations, use is made of the Integrated Noise Model (INM) version 7.0b. The INM has been the FAA's standard tool for airport noise assessment for more than 3 decades, and is probably the most widely used noise assessment tool throughout the world. To model the cumulative noise impact, the cumulative noise level $L_{d e n}$ has been introduced. $L_{d e n}$ is a day-evening-night noise metric which takes the total amount of noise produced into account over a 24 hour period. The $L_{d e n}$ relation is given as follow [8], [9]:

$L_{\text {den }}=10 \log _{10}\left[\sum_{i=1}^{N_{A / C \text { t tpes }}} n(i) \cdot \omega(i) \cdot 10^{\frac{S E L(i)}{10}}\right]-10 \log _{10} T_{1}$,

where $i$ represents the $i^{\text {th }}$ aircraft type under consideration, $n(i)$ is the number of movements of that particular type, $\omega(i)$ is the average(day/night/evening) penalty factors over a 24 hour period for the $i^{\text {th }}$ aircraft type, and $\operatorname{SEL}(i)$ is the Sound Exposure Level for the $i^{\text {th }}$ aircraft type. Finally, the time factor $T_{1}$ represents the averaging period in seconds, $24 \cdot 3600$ seconds.

The final step in assessing the community noise impact lies in determining the total number of people that can be considered to experience sleep disturbance due to aviation noise in the area surrounding the airport. Next, a dose-response relationship is applied which couples the local $L_{d e n}$ levels to the local population. This dose-response relationship, developed by Dutch research organizations TNO and RIVM (National Institute for Public Health), is based on a large-scale research effort in 2002, and is considered to be the standard relationship in The Netherlands . which can be expressed as:

$$
\% P H=\left(\frac{e^{-8.11001+0.1333 L_{d e n}}}{1+e^{-8.11001+0.1333 L_{d e n}}}\right) \cdot 100
$$

where $\% P H$ represents the percentage of people highly annoyed by aircraft noise due to a certain $L_{d e n}$ value. By determining $\% P H$ for each grid cell in the GIS and multiplying with the number of people living in that cell, the total number of people highly annoyed, $P H$, can be determined. This then serves as the environmental performance criterion in the trajectory optimization.

Although the main objective of this study is to minimize the total community noise impact of routes, to account for airline economic interests and local air quality considerations, the total path length was also added as an optimization criterion. The composite optimization criterion for this study then becomes: 


$$
J=s_{f}+k_{\text {noise }} P H
$$

where $s_{f}$ is the total trajectory distance, and $k_{\text {noise }}$ is a weighting factor. By parametrically varying this weighting factor, the relative weight of the number of community noise impact in the optimization problem can be changed.

\section{OPTIMIZATION FRAMEWORK}

\section{A. Rational Bezier Curve}

Rational Bezier curve [10] is a parametric curve frequently used in computer graphics and related fields. The equation of a rational Bezier curve is

$$
\mathrm{P}(\omega)=\frac{\sum_{i=0}^{n} \lambda_{i} B_{i}^{n}(\omega) \mathbf{b}_{i}}{\sum_{i=0}^{n} \lambda_{i} B_{i}^{n}(\omega)}
$$

where $\omega \in[0,1]$ is free parameter, $\lambda_{i}$ is a weight, $b_{i}$ is a control point and $B_{n, i}(\omega)$ are Bernstein polynomials, defined explicitly as

$$
B_{n, i}(\omega)=\left(\begin{array}{c}
n \\
i
\end{array}\right) \omega^{i}(1-\omega)^{n-i}
$$

The recursive algorithm for calculating the rational Bezier curve is formulated as

$$
P(\omega)=\frac{\sum_{i=0}^{n} \lambda_{i}^{k} \mathbf{b}_{i}^{k} B_{i}^{n-k}(\omega)}{\sum_{i=0}^{n} \lambda_{i}^{k} B_{i}^{n-k}(\omega)}=\cdots=\boldsymbol{b}_{0}^{n}
$$

where the intermediate weights $\lambda_{i}^{k}$ and intermediate points are defined as

$$
\begin{aligned}
& \lambda_{i}^{k}=\left\{\begin{array}{lc}
\lambda_{i}, & k=0 \\
(1-\omega) \lambda_{i}^{k-1}+\omega \lambda_{i+1}^{k}, & k=1,2, \cdots n ; i=0,1, \cdots, n-k
\end{array}\right. \\
& \boldsymbol{b}_{i}^{k}=\left\{\begin{array}{lc}
\boldsymbol{b}_{i}, & k=0 \\
(1-\omega) \frac{\lambda_{i}^{k-1}}{\lambda_{i}^{k}} \boldsymbol{b}_{i}^{k-1}+\omega \frac{\lambda_{i+1}^{k-1}}{\lambda_{i}^{k}} \boldsymbol{b}_{i+1}^{k-1}, & k=1,2, \cdots, n ;
\end{array}\right. \\
&
\end{aligned}
$$

Denoting

$$
P^{*}(\omega)=\sum_{i=0}^{n} \lambda_{i} \boldsymbol{b}_{i} B_{i}^{n}(\omega), \lambda(\omega)=\sum_{i=0}^{n} \lambda_{i} B_{i}^{n}(\omega)
$$
as

Then the derivatives of Rational Bezier Curve can be given

$$
P^{(k)}(\omega)=\frac{1}{\lambda(\omega)}\left[P^{*(k)}(\omega)-\sum_{j=1}^{k}\left(\begin{array}{l}
j \\
k
\end{array}\right) \lambda^{(j)}(\omega) P^{(k-j)}(\omega)\right]
$$

Additionally, $P^{*}(\omega) \quad, \quad P^{\prime}(\omega) \quad, \quad P^{\prime \prime}(\omega) \cdots \quad$ and $\lambda(\omega), \lambda^{\prime}(\omega), \lambda^{\prime \prime}(\omega) \cdots$ can be calculated by de Casteljau algorithm [10].

\section{B. Ground Track Shaping Based on Rational Bezier Curve}

The aircraft ground track can be parameterized by the rational Bezier curve as

$$
\boldsymbol{P}(\Gamma, \omega)=[x(\Gamma, \omega), y(\Gamma, \omega)]^{T}
$$

where parameter $\omega \in[0,1]$, and $\Gamma=\left\{\tau_{i} \mid i=1,2, \cdots, q\right\}$ is a set of free parameters, constitutes of control points and weights. Given the free parameters $\tau_{i}$, the ground track is determined and the heading angle and its derivative along the resulting ground track can be formulated as follows (where prime denotes differentiation with respect $\omega$ ):

$$
\begin{gathered}
\tan \chi=\frac{x^{\prime}}{y^{\prime}} \Rightarrow \chi=\arctan \left(\frac{x^{\prime}}{y^{\prime}}\right) \\
\chi^{\prime}=\frac{x^{\prime \prime} y^{\prime}-x^{\prime} y^{\prime \prime}}{y^{\prime 2}} \cos ^{2} \chi
\end{gathered}
$$

Noting that

$$
\begin{gathered}
\frac{d s}{d \omega}=\sqrt{x^{\prime 2}+y^{\prime 2}} \Rightarrow \frac{d \omega}{d s}=\frac{1}{\sqrt{x^{\prime 2}+y^{\prime 2}}} \\
V(s)=\frac{d s}{d t} \Rightarrow \Delta t=\frac{1}{V(s)} \Delta s \\
\frac{d y}{d s}=\frac{d y}{d \omega} \frac{d \omega}{d s}=\cos \chi
\end{gathered}
$$

We have

$$
\begin{gathered}
u(s)=\frac{d \chi}{d s}=\frac{d \chi}{d \omega} \frac{d \omega}{d s}=\frac{x^{\prime \prime} y^{\prime}-x^{\prime} y^{\prime \prime}}{\left(x^{\prime 2}+y^{\prime 2}\right)^{3 / 2}} \\
\tan \mu(s)=u(s) \frac{V(s)^{2}}{g_{0}} \\
C_{L}=\frac{2 W}{\rho V^{2}(s) S} \frac{1}{\cos \mu(s)} \\
T(s)=V(s) \frac{d V(s)}{d s} \frac{W}{g_{0}}+\frac{1}{2} \rho(s) V(s)^{2} S C_{D}+W \frac{d h(s)}{d s}
\end{gathered}
$$

Consequently, the sound exposure levels ( $S E L$ ) at specified location around the airport can be calculated according to the thrust $T(s)$. Therefore, the composite performance index

$$
J=k_{\text {noise }} P H+k_{\text {distance }} s_{f}
$$

can be determined by using the following functions:

$$
\begin{gathered}
L_{\mathrm{den}}=10 \log \left[\sum_{i=1}^{N_{A C \text { trpes }}} n(i) \cdot w(i) 10^{\frac{S E L(i)}{10}}\right]-10 \log T_{1}(d B A) \\
\% P H=\left(\frac{e^{-8.11001+0.1333 L_{\mathrm{den}}}}{1+e^{-8.11001+0.1333 L_{\mathrm{den}}}}\right) \cdot 100 \\
s_{f}=\int_{0}^{1} \sqrt{x^{\prime 2}+y^{\prime 2}} d \omega
\end{gathered}
$$

where $T_{1}$ is equal to $24 \cdot 3600$.

Finally, the trajectory optimization problem can be reduced to a parameter optimization problem as follows:

Determine the optimal parameters $\tau_{i}^{*}$ that minimize the performance index

$$
J=k_{\text {noise }} P H+k_{\text {distance }} \int_{0}^{1} \sqrt{x^{\prime 2}+y^{\prime 2}} d \omega
$$


Subject to the boundary conditions

$$
\begin{aligned}
& \phi\left(0, s_{0}, x\left(s_{0}\right), y\left(s_{0}\right), \chi\left(s_{0}\right)\right)=0 \\
& \varphi\left(1, s_{f}, x\left(s_{f}\right), y\left(s_{f}\right), \chi\left(s_{f}\right)\right)=0
\end{aligned}
$$

Maximum rate of turn constraints

$$
\left|\dot{\chi}_{s}\right| \cdot V_{\max }(s(\omega)) \leq c \Leftrightarrow\left|\dot{\chi}_{s}\right| \leq \frac{c}{V_{\max }(s(\omega))}
$$

and maximum angle of bank constraints

$$
|\mu| \leq \mu_{\max } \Leftrightarrow \tan ^{-1}\left(\frac{1}{g_{0}}\left|\dot{\chi}_{s}\right| V_{\max }^{2}(s(\omega))\right) \leq \mu_{\max }
$$

where $s(\omega)=\int_{0}^{\omega} \sqrt{x^{\prime 2}+y^{\prime 2}} d \zeta, x=x(\Gamma, \omega), y=y(\Gamma, \omega)$, $\omega \in[0,1], \Gamma=\left\{\tau_{i} \mid i=1,2, \cdots, q\right\}, V(s(\omega))$ is prescribed.

For the simultaneous routing optimization, there are several routings involved in the optimization, and none of them are allowed to intersect with each other. Therefore, non-intersecting constraints need to be imposed.

\section{Data-Points Selection}

In this study, the boundary constraints, such as position and heading angle, are satisfied automatically due to the properties of the rational Bezier curves. In order to handle the remaining constraints, the ground track is discretized into many data points $\boldsymbol{P}\left(\Gamma, \omega_{i}\right), \omega_{i} \in[0,1]$, and approximated by line segments. The maximum rate of turn and maximum angle of bank constraints are guaranteed at the data points. It is noted that two original tracks will be non-intersecting if one's piecewise linear approximated curve doesn't intersect with the other's. It also should be noted that this is a necessary condition for non-intersection, but not a sufficient one, finer data points contribute to better approximation and higher precision, but require much more calculating time, and will be inefficient. Therefore, only the most significant points are needed in the piecewise linear curve approximation. An obvious choice to measure the significance of the single data point is the absolute curvature at that point [11]. In this study, a data-points selection method based on absolute curvature is formulated.

Having shaped the ground track by rational Bezier curve, the absolute curvature $k_{i}$ and point $P_{i}=\left[x_{i}, y_{i}\right]$ are known for each $\omega_{i}, i=1, \cdots m$. The next step is the selection of a specified number of nodes $\omega_{i}$ based on the absolute curvature $k_{i}$. This is done in such a way that the curvature is distributed uniformly with respect to chord length. This step is explained next.

The chord length of the piecewise linear curve implied by $P_{i}=\left[x_{i}, y_{i}\right], i=0, \cdots m$ is defined as

$$
S=\sum_{i=0}^{m-1} S_{i}
$$

where $s_{i}=\left\|P_{i+1}-P_{i}\right\|$. Integrating the absolute curvature function $k_{i}$ yields

$$
K=\int_{0}^{S} k d s=\frac{1}{2} \sum_{i=0}^{m-1} s_{i}\left(k_{i}+k_{i+1}\right)
$$

By rearranging the summation in (28) one obtains

$$
K=\frac{1}{2} k_{0} s_{0}+\frac{1}{2} \sum_{i=1}^{m-1} k_{i}\left(s_{i-1}+s_{i}\right)+\frac{1}{2} k_{m} s_{m-1}
$$

Formula (29) emphasizes that the integrated absolute curvature estimate $k_{i}$ multiplies the length of the line segments on both sides, $s_{i-1}$ and $s_{i}$. Consequently, greatest weight is given to data points with high absolute curvature and long line segments on both sides, while least weight is given to data points with low absolute curvature and short line segments on both sides. Therefore, the significant data points can be selected such that the integrated absolute curvature estimate is distributed nearly uniformly with respect to chord length. Assuming that $K>0$, one computes $l+1$ values $\bar{k}_{j}$ such that

$$
\bar{k}_{j}=j \frac{K}{l}, j=0, \cdots, l
$$

Let

$$
\begin{gathered}
\Xi=\left\{\theta_{i} \| \theta_{i}=\frac{1}{2} \sum_{e=0}^{i-1} s_{e}\left(k_{e}+k_{e+1}\right), e=0, \cdots, m\right\} \\
\Psi=\left\{\omega_{i} \| \omega_{i} \in[0,1], i=0, \cdots, m\right\}
\end{gathered}
$$

By making use of the monotone property of $\theta_{i}, i=0, \cdots, m$, nodes $\bar{\omega}_{j}$ according to $\bar{k}_{j}, j=0, \cdots, l$ can be determined through interpolation. Finally, the data points $\bar{P}_{j}=\left[x_{j}, y_{j}\right], j=0, \cdots l$ corresponding to the selected nodes $\bar{\omega}_{j}, j=0, \cdots, l$ are the significant data points. The selected data points distributed non-uniformly along the track according to the characteristic of the curvature and the resulting piecewise linear curves can perform a better approximation comparing to the uniform counterpart. Additionally, because the selected points are most likely to violate the maximum rate of turn and maximum angle of bank, if the track obeys the constraints on these points, the constraints will not be violated along the track theoretically, namely the feasibility of the track could be guaranteed.

\section{Constraints Handing}

Given the selected nodes $\bar{\omega}_{j}, j=0, \cdots, l$, penalty function is introduced to handle the constraints. The penalty function can be formulated as follows:

$$
p=p_{\dot{\lambda}_{s}}+p_{\mu}+p_{\text {ints }}
$$

where $p_{\text {ints }}$ is the integration of the difference in $x$ direction, and

$$
\begin{gathered}
p_{\dot{\lambda}_{s}}=\sum_{i=0}^{l} \max _{i}\left\{\left|\dot{\chi}_{s}\right|_{i}-\frac{c}{V_{\max }\left(s\left(\omega_{i}\right)\right)}, 0\right\} \\
p_{\mu}=\sum_{i=0}^{l} \max _{i}\left\{|\mu|_{i}-\mu_{\max }, 0\right\} \\
\left|\dot{\chi}_{s}\right|_{i}=\left|\dot{\chi}_{s}\left(\bar{\omega}_{i}\right)\right|,|\mu|_{i}=\left|\mu\left(\bar{\omega}_{i}\right)\right| .
\end{gathered}
$$




\section{NUMERICAL EXAMPLE}

The simultaneous optimization of the departure routes for the Kaagbaan and Aalsmbeerbaan runways South-outbound is present in this study. A genetic algorithm and pattern search method are used to search the optimal solution. The optimal solution is searched by the genetic algorithm and refined by the pattern search method. The constraints are given as follows:

1) Start and end point of the track (Table I):

TABLE I: START AND END POINT OF THE TRACK

\begin{tabular}{|l|l|l|l|c|}
\hline \multicolumn{4}{|c|}{ Start point $(\mathrm{km})$} & \multicolumn{2}{l|}{ End point $(\mathrm{km})$} \\
\hline Runway & $X_{\text {DER }}$ & $Y_{\text {DER }}$ & $X_{\text {DER }}$ & $y_{\text {DER }}$ \\
\hline Kaagbaan & -1.5546 & -2.1217 & 2.698 & -40.0 \\
& & & 8 & \\
\hline Aalsmeerbaan & 1.1518 & -1.7906 & 2.710 & -40.0 \\
& & & 4 & \\
\hline
\end{tabular}

2) Maximum rate of turn:

$$
\left|\dot{\chi}_{s}\right| \leq \frac{3}{V_{\max }(s(\omega))} \frac{\pi}{180}
$$

3) Maximum angle of bank:

$$
\left|\dot{\chi}_{s}\right| \leq \frac{g_{0} \cdot \tan \left(\frac{d \cdot \pi}{180}\right)}{V_{\max }(s(\omega))^{2}}
$$

where

$$
d= \begin{cases}15^{\circ} & , h \leq 304.8 \mathrm{~m} \\ 20^{\circ} & , 304.8<h \leq 914.4 \mathrm{~m} \\ 25^{\circ} & , h>914.4 \mathrm{~m}\end{cases}
$$

In order to improve the searching performance, the augmented performance index is scaled as follow:

$$
J=k_{\text {noise }} \frac{P H}{e_{1}}+k_{\text {distance }} \frac{s_{f}}{e_{2}}+\rho\left(k_{\dot{\lambda}_{s}} p_{\dot{\lambda}_{s}}+k_{\mu} p_{\mu}+k_{\text {ints }} \frac{p_{\text {ints }}}{e_{3}}\right)
$$

where $e_{1}, e_{2}$ and $e_{3}$ are user-defined positive constants that scale the amount of constraint violation. In this study, $e_{1}=e_{2}=1 \times 10^{4}, e_{3}=1 \times 10^{5}$. The parameter $\rho$ is updated at every generation $g$ in the following way:

$$
\rho=1+\left(\frac{g}{g_{\max }}\right)^{2}
$$

where $g_{\max }$ is the maximum number of generations.

In simultaneous optimization, $p_{\dot{\lambda}_{s}}$ and $p_{\mu}$ allow for total constraints violation of routes involved, and

$$
s_{f}=\frac{1}{2}\left(s_{f, \text { Aalsmbeerbaan }}+s_{f, \text { Kaagbaan }}\right)
$$

Two solutions are obtained in this study. The first one is for $k_{\text {noise }}=1, k_{\text {distance }}=1, k_{\dot{\lambda}_{s}}=0.01, k_{\mu}=2$ and $k_{\text {ints }}=4$. Fig. 1 and Fig. 2 present the simultaneously and separately optimized routes on a map, respectively. They are obtained using the same cost indices. From Fig. 1 it can be seen that the routes in separate optimization are intersecting with each other. This is not valid in airport operation. In contrast, the simultaneously optimized route for Kaagbaan features a wide initial turn and approaches to but does not cross the optimized route for Aalsmeerbaan, as shown in Fig. 2. Obviously, the initial turn is to avoid overflying the densely populated area. From Table II it can be seen that the simultaneous optimization does perform better than the benchmark in both number of people highly annoyed and track distance. The solution in simultaneous optimization reveals a $4.70 \%$ and $4.77 \%$ decrease in the number of people highly annoyed and track distance relative to the benchmark scenario. In Fig. 3 and Fig. 4 the data-points selection for Kaagbaan and Aalsmeerbaan South-outbound departure route in simultaneous optimization respectively are presented. It can be seen that the selected points are always located at the non-smooth part of the routes. These points can capture the key characteristics of the original route, and the resulting piecewise linear curve could approximate the original track well.

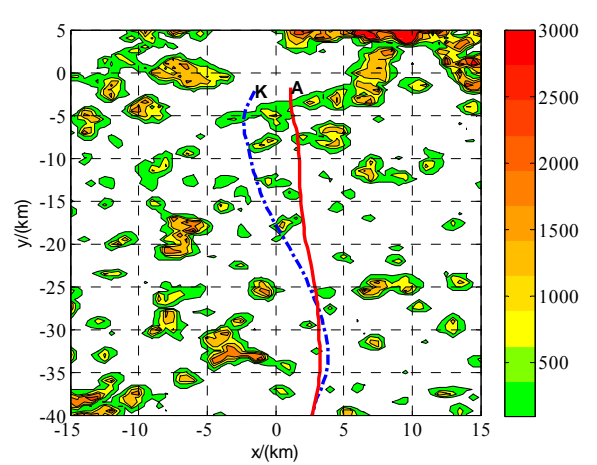

Fig. 1. Routes for separate optimization.

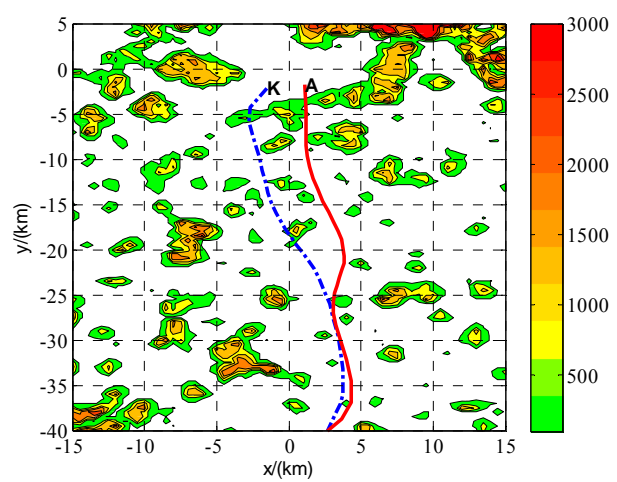

Fig. 2. Routes for Simultaneous optimization.

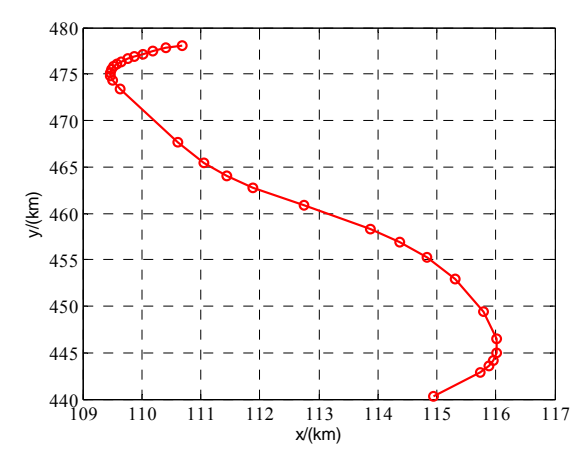

Fig. 3. Data-points distribution(Kaagbaan).

Similar results are shown in Fig. 5, Fig. 6 and Table III for $k_{\text {noise }}=2, k_{\text {distance }}=1, k_{\dot{\lambda}_{s}}=0.01, k_{\mu}=3$ and $k_{\text {ints }}=3$. The solution in simultaneous optimization reveals a $7.21 \%$ and 
$1.51 \%$ decrease in the number of people highly annoyed and track distance relative to the benchmark scenario. It is clear that, compared with the former case, this solution tends to decrease the number of people highly annoyed at the expense of increasing the total track distance.

A. Optimized Route for $k_{\text {noise }}=1, k_{\text {distance }}=1$

TABLE II: OPTIMIZATION RESULTS A
\begin{tabular}{|c|c|c|c|c|}
\hline & \multicolumn{3}{|c|}{ Benchmark } & Simultaneous \\
\hline & Aals & Kaag & Aals & Kaag \\
\hline \multirow{2}{*}{ Distance (m) } & 3996 & 4231 & 3913 & 3922 \\
& 6 & 5 & 1 & 9 \\
\hline People highly annoyed & \multicolumn{2}{|c|}{107441} & \multicolumn{2}{c|}{102394} \\
\hline
\end{tabular}

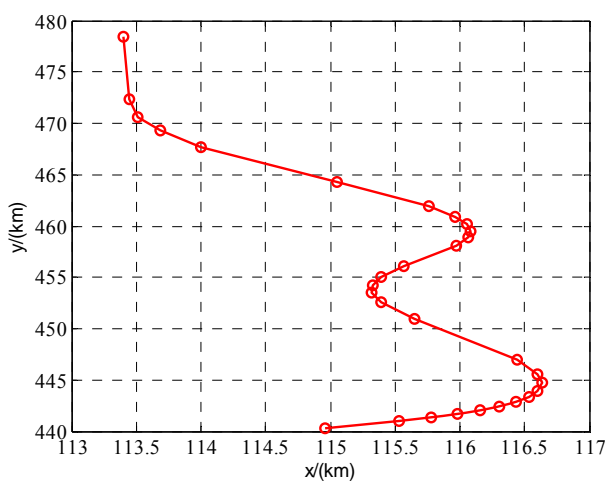

Fig. 4. Data-points distribution(Aalsmeerbaan).

B. Optimized Route for $k_{\text {noise }}=2, k_{\text {distance }}=1$

TABLE III: OPTIMIZATION RESULTS B

\begin{tabular}{|c|c|c|c|c|}
\hline & \multicolumn{2}{|c|}{ Benchmark } & \multicolumn{2}{c|}{ Simultaneous } \\
\hline & Aals & Kaag & Aals & Kaag \\
\hline \multirow{2}{*}{ Distance (m) } & 3996 & 4231 & 3887 & 4216 \\
& 6 & 5 & 6 & 5 \\
\hline People highly annoyed & \multicolumn{3}{|c|}{107441} & \multicolumn{2}{c|}{99693} \\
\hline
\end{tabular}

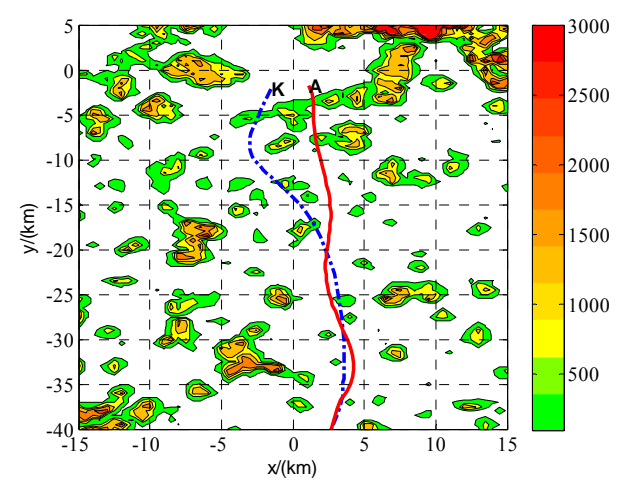

Fig. 5. Routes in separated optimization.

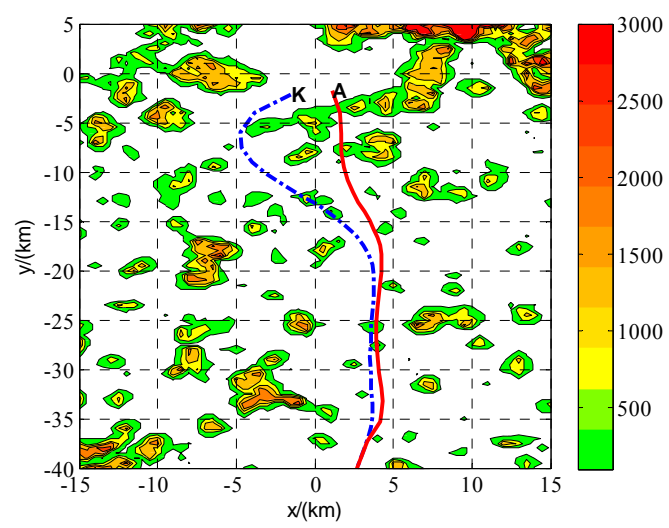

Fig. 6. Routes in simultaneous optimization.

\section{CONCLUSION}

Through the course of this research, we have developed a novel methodology to optimally shape the shared ground tracks under given procedures for the various aircraft types that visit Amsterdam Airport Schiphol within a specified period. An important conclusion of this research is that the proposed methodology is capable of optimizing departure routes simultaneously with respect to noise and track length, taking into account the multiple aircraft types that are present in the traffic mix. It is proved that the simultaneous optimization does perform better than the benchmark. Moreover, the optimized simultaneous routes tend to get close to each other in order to decrease the total noise level of near-airport region.

\section{REFERENCES}

[1] Amsterdam Airport Schiphol. (2011). [Online]. Available: http://www.schiphol.nl.

[2] L. J. J. Erkelens, "Advanced noise abatement procedures for approach and departure," in Proc. the AIAA Guidance, Navigation and Control Conference and Exhibit, pp. 5-8, 2002.

[3] X. Prats, V. Puig et al., "Optimal departure aircraft trajectories minimising population annoyance," in Proc. the $3^{\text {rd }}$ International Conference on Research in Air Transportation ICRAT, pp. 1-4, 2008.

[4] H. G. Visser and R. A. A. Wijnen, "Optimisation of Noise Abatement Arrival Trajectories," The Aeronautical Journal, vol. 107, 2003, pp. 607-615.

[5] H. G. Visser, "Generic and site-specific criteria in the optimization of noise abatement trajectories," Transportation Research Part D: Transport and Environment, vol. 10, no. 5, 2005, pp. 405-419.

[6] H. G. Visser and R. A. A. Wijnen, "Optimization of noise abatement departure trajectories," Journal of Aircraft, vol. 38, no. 4, 2001, pp. 620-627.

[7] R. A. A. Wijnen and H. G. Visser, "Optimal departure trajectories with respect to sleep disturbance," Aerospace Science and Technology, vol. 7, pp. 81-91, January 2003

[8] J. Dons, "Optimization of departure and arrival routing for amsterdam airport schiphol," Master of sciene thesis, Faculty of Aerospace Engineering, Delft University of Technology, June, 2012.

[9] M. L. Braakenburg, "Development of a multi-event optimization tool for noise optimized arrival route design," Master of sciene thesis, Faculty of Aerospace Engineering, Delft University of Technology, October 2010.

[10] F. Z. Shi, Rational b-Spline Curve and Surface II, CAGD \& NURBS, Beijing: Higher Education Press, 2001, ch. 10, pp. 382-393.

[11] B. Hamann and J. L. Chen, "Data point selection for piecewise linear curve approximation," Computer Aided Geometric Design, vol. 11, no. 3, 1994, pp. 289-301.

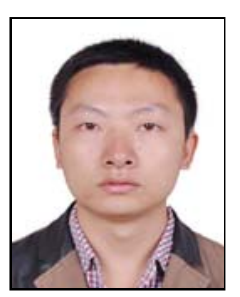

Zhang Song was born on October 11, 1985, in Guizhou province, China. He received his B.S.E. degree and M. S. E. from Northwestern Polytechnical University (NPU) in 2009 and 2011. $\mathrm{He}$ is pursuing a Ph.D. from NPU. Mr. Zhang joined air transport and operations at Delft University of Technology in September 2013 and he as a visiting researcher and currently conducts work on noise abatement route design in airport operation. His interests include trajectory optimization and guidance for flight vehicles.

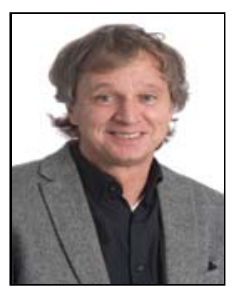

Hendrikus Gerrit Visser was born on September 22, 1957, in Netherland. He received his engineering degrees in aeronautics from Delft University of Technology, Delft, The Netherlands in 1981 and 1982, respectively, and a Ph.D. in aerospace and ocean engineering from Virginia Polytechnic Institute and State University in 1985.

In 1983 he was a visiting research associate at the Israel Institute of Technology, Haifa, Israel. From 1985 to 1987 he worked as a system engineer at Fokker Space \& Systems, Amsterdam, Netherlands. In 1987 he joined the Delft University of Technology, where he is currently an associate professor at the Faculty 
of Aerospace Engineering. His interests include flight operations, air traffic control, flight mechanics and optimization theory. He is an Associate Fellow of the AIAA.

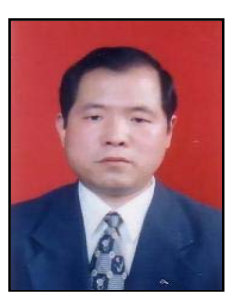

Hou Mingshan was born in December, 1957, in Shan'xi province, China. He received his engineering degrees in guidance, navigation and control from Northwestern Polytechnical University(NPU), Shan'xi province, China in 1983, 1986 and 1989, respectively. In 1989 he joined the NPU, where he is currently a professor in the School of Automation. His interests include advanced guidance, control theory and application, complex system control, trajectory optimization et al.

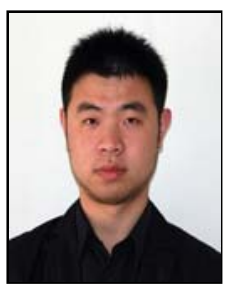

Cong Wei was born in December 24, 1988, in Jiangsu province, China. He received his B.S.E. degree from Nanjing University of Aeronautics and Astronauticsm (NUAA) in 2011. He is pursuing a Ph.D. from NUAA. Mr. Cong joined Air Transport and Operations at Delft University of Technology in May 2014 as a visiting researcher and currently conducts work on safety assessment in air traffic operation. His interests include air traffic complexity and human dynamics. 